\title{
Design and Experiment of Precision Flatness Measurement Device for Miniature Parts
}

\author{
Yu Zhang ${ }^{1, a^{*}}$, Hongxi Wang ${ }^{2}$, Li Feng ${ }^{3}$, Yaning $\mathrm{Li}^{3}$ \\ ${ }^{1}$ School of Mechatronic Engineering, Xi'an Technological University, Xi'an 710032, China \\ a1289570740@qq.com
}

Keywords: Precision measurement; Flexure hinge; Flatness; Miniature parts

\begin{abstract}
To achieve stringent evaluation of the flatness error for some miniature parts, a novel flatness measurement device based on the coordinate measurement method is designed. A two-dimensional flexible guide mechanism is used as the flatness measurement datum. The micrometer head drives the flexible guide way to accomplish two-dimensional scanning movement in plane. An inductive measurement head whose measuring force is adjustable is used to measure work piece surface. A sample is measured in the range of $10 \mathrm{~mm} \times 10 \mathrm{~mm}$ and the flatness error $0.48 \mu \mathrm{m}$ is obtained with the evaluation of the least square method.
\end{abstract}

\section{Introduction}

With the rapid development of precision and ultra-precision manufacturing technology and equipment [1], and the flatness of some parts is higher and higher, especially in some small parts, such as static pressure bearing assembly for aerospace, hydrostatic guideway in floating components, semiconductor wafer, precision gauge etc.. These parts are all characterized with high flatness precision (sub-micron level) and small plane size, which bring the challenge to the precision measurement of the flatness.

The main instruments of the flatness measurement include gradienter,autocollimator, dial gauge, three coordinate measuring machine, optical flat and so on [2]. The autocollimator, gradient for measurement of large size parts. The human factors of measurement with micrometer and dial indicator is larger and the measuring precision fails to meet requirements. The measurement accuracy of three-coordinate measuring machine (CMM) is generally difficult to achieve $1 \mu \mathrm{m}$ or less, thus it's not suitable for measuring flatness of small parts. An optical flat is commonly used measuring flatness of small parts at present, but the influence of human factors on the flatness error is larger. In recent years, the nano-coordinate measuring machines (NPM) [3-4] has already been invented. The equipment can realize the precision measurement of small flat, but NPM is expensive, therefore it is only used for laboratory currently.

In view of the existing difficulties of micro parts in flatness measurement, the literature called application of precision turntable in flatness measurement [5], which was combined with autocollimator and obtained the uncertainty of $5 \mu \mathrm{m}$ of accuracy in the flatness measurement. A new method for measuring flatness error by using a profiler is used in the literature [6] and the degree of $60 \mathrm{~mm} * 60 \mathrm{~mm}$ plane is measured by using a profile meter. The literature called a new method for measuring flatness error by using the cylindrical degree instrument [7], which achieves flatness measurement by using roundness instrument. Although the measurement of flatness can be realized with the aid of the existing precision instruments, the measurement process and data processing are still complex, and the measurement accuracy and repeatability cannot be guaranteed.

This paper designed the flatness precision measuring device of small parts, the device adopts a parallelogram flexible guiding mechanism, which achieves two-dimensional scanning motion manually and reads the height value of sample surface through inductive probe. It's measuring principle is the same as the CMM and the experiments in the preliminary of testing the planeness show that the precision of measuring device can reach sub-micron. 


\section{Flatness Measuring Device Design}

The core of the contact-type flatness measuring device is a precision guide rail, the traditional guide rails mainly include slide guide rail, rolling guide rail and static pressure guide rail. The oriented precision of slide guide and rolling guide can be achieved in micron level (the specific accuracy relates with stroke). Both the slide guide rail and rolling guide rail are characterized with simple structure, but the accuracy is not high. Hydrostatic guide rail mainly include liquid static pressure guide rail and gas static pressure guide rail. Hydrostatic guide way has the characteristics of high precision and high stiffness, and it is widely used in the field of ultra-precision machining [8]. Gas static pressure guide rail is the standard configuration of the three coordinate measuring machines (CMM), the technology is mature and the precision is high. Hydrostatic guide rail need accessory equipment, which can provide hydraulic or pneumatic, thus the structure of system is complex and the cost is high.

The rail based on flexible hinges (or guiding mechanism) is a kind of guide way in the field of ultra-precision machining and measurement in recent years, it has been the important application in aerospace, optical instruments, precision measurement and bio engineering and other fields [9]. The flexible hinge is used as the kinematic pair of the mechanism, and the elastic deformation of the material is adopted to realize the micro equivalent motion, so that the error caused by the joint can be minimized. It is characterized by compact structure, small size, and it can be done without mechanical friction, no gap, no need for lubrication, it still has a high displacement resolution [10], so it is a more ideal guide mechanism for precision machinery.

The travels of precision flexible steering mechanism are within $50 \mu \mathrm{m}$ [11], it cannot meet the requirements of the travel guide mechanism for flatness measurement. In order to increase the stroke, this paper adopts the design of a parallel quadrilateral guiding mechanism, as shown in Fig. 1 , the flexible hinge spring instead of integration process to avoid fatigue damage due to excessive deformation. Under the effect of thrust $\mathrm{F}$, the guiding mechanism generates displacement of $\Delta x$ in $\mathrm{X}$ direction, thus causes displacement of $\Delta \mathrm{y}$ in $\mathrm{Y}$ direction, theoretically $\Delta \mathrm{y}$ is in the xoy plane, so $\mathrm{X}$ direction can be the scanning direction of motion measurement, and the $\mathrm{Z}$ direction as be the direction of measurement. Flatness measurement requires two-dimensional scanning motion, as shown in Fig. 2, the 2 sets of flexible guide mechanisms have been nested, thus you can achieve the two-dimensional scanning motion in the xoy plane.

The motion accuracy of two-dimensional table is the core of the flatness measurement level, two-dimensional flexible guide mechanism as have been shown in Fig. 2, $\mathrm{x}$ and $\mathrm{y}$ are coupled in the direction of the movement, but theoretically this kind of coupling is in the xoy plane, it does not affect the accuracy in the $\mathrm{Z}$ direction, which is the theoretical basis of the flatness measurement with the structure.

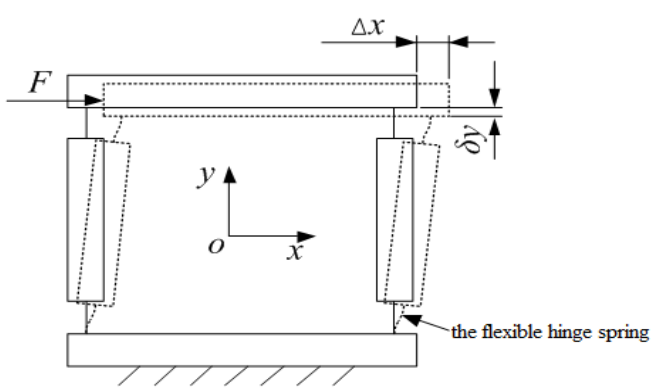

Figure 1. Schematic diagram of one dimensional flexible guide structure

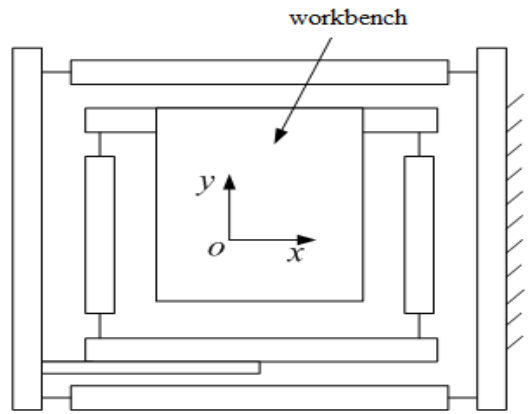

Figure 2. Schematic diagram of two dimensional guide rail

Based on the above analysis, the overall structure of the flatness measurement device is designed as shown in Fig. 3. The measuring device is mainly composed of two parts, one is the realization of two-dimensional planar motion mechanism, and the other is the detection of micro displacement. The two-dimensional motion mechanism is composed of a two-dimensional flexible guide mechanism, differential head traction steel wire driving mechanism, a workbench and a work piece 
fixture. Micro displacement measurement is mainly composed of LVDT sensor, measuring needle, force adjusting mechanism and a detection circuit.

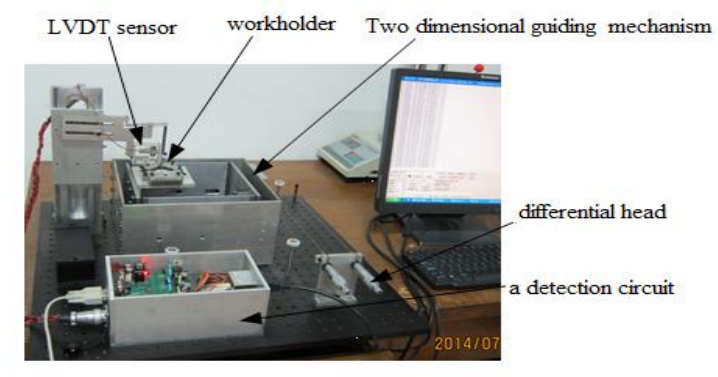

Figure 3. The precision flatness measurement device for tiny parts

Fig. 4 and Fig. 5 are micro-displacement detection circuit schematic and physical map. Displacement sensor uses MHR050 type LVDT sensor, it's linear range is $\pm 1.27 \mathrm{~mm}$, and it can realize the resolution in the linear range of $0.02 \mu \mathrm{m}$.

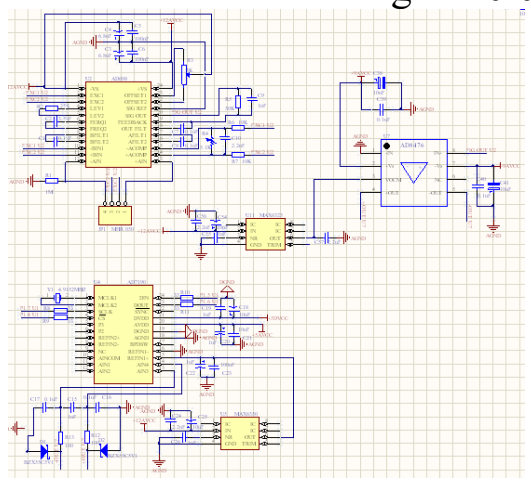

Figure 4. Schematic diagram of displacement detection circuit

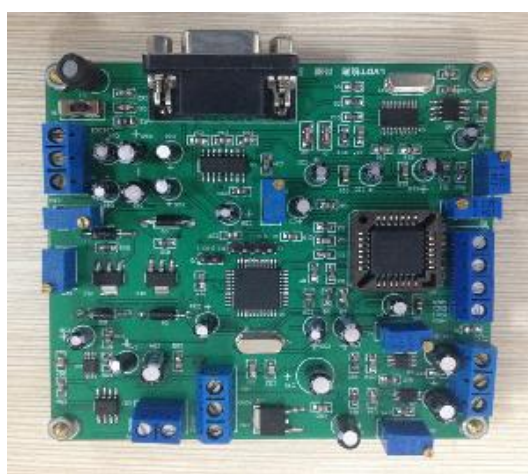

Figure 5. Displacement detection circuit

\section{Experimental Testing}

In order to test the accuracy of the designed flatness measuring device, as shown in Fig. 6, the test sample whose diameter is $24 \mathrm{~mm}$ has been cut from the hard disk, and then the sample has been regarded as a reference plane, in order to test the accuracy initially. The step size of $X$ direction and $\mathrm{Y}$ direction is $1 \mathrm{~mm}$, and through the detection circuit we can read the height change data of the $\mathrm{Z}$ direction in the range of $10 \mathrm{~mm} \times 10 \mathrm{~mm}$.

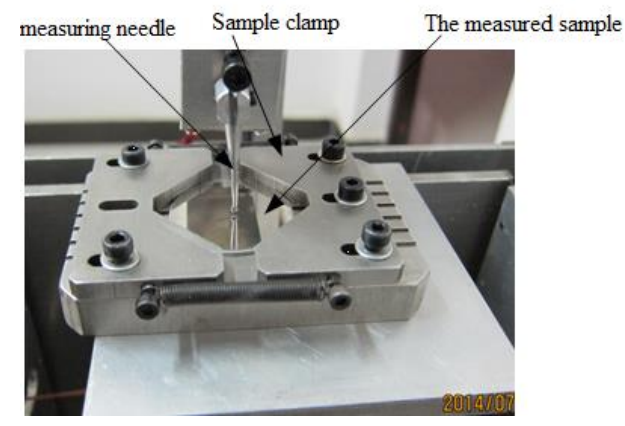

Figure 6. The measured sample installation

The test data is drawn into a three-dimensional surface, as shown in Fig. 7, from the graph it can be seen there is an angle between the measured parts and the guiding plane. In order to facilitate the observation, the measured data is subtracted from the minimum value, and then according to the rotation of $\mathrm{X}$ axis and $\mathrm{Y}$ axis direction, thus we can get the three-dimensional surface measurement 
of the plane as shown in Fig. 8.

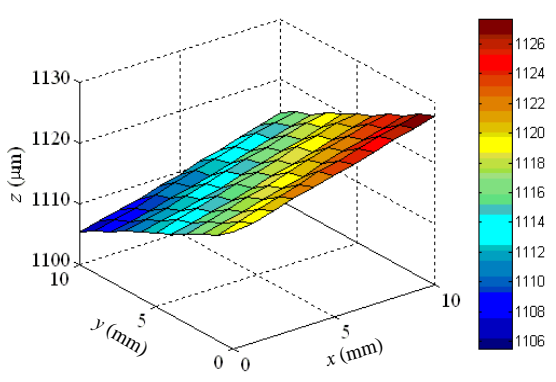

Figure 7. The original measurement results of sample surface

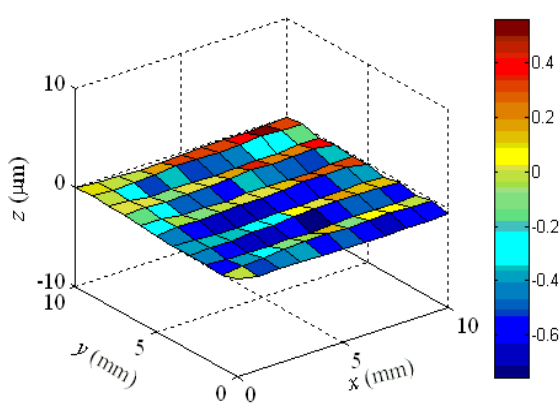

Figure 8. The sample surface measurement results after the coordinate transformation

The flatness error is the variation of the measured plane to the ideal surface, and the method of evaluation includes three far points method, the least square method, and minimum inclusive area method. The least square method is used to evaluate the flatness error in the following part.

The coordinate value of the measured point in the measured plane is $P_{i, j}\left(x_{i}, y_{j}, z_{i, j}\right)$. The plane equation (refer with Eq. 1) is established in three dimensional space coordinate system is:

$$
z^{*}=a x+b y+c
$$

Where, $\mathrm{a}, \mathrm{b}, \mathrm{c}$ are the undetermined coefficient and $\mathrm{z}^{*}$ is the ideal coordinate value of the measured point.

In order to minimize the sum of the squares of the variation of each measurement point with respect to the plane (evaluation plane), the function is as follow:

$\phi a(b, c) \quad \sum\left(z_{i, j} \quad z_{i, j}^{*}\right)^{2} \quad \sum\left(z_{i, j} \quad a x_{i}-b x_{j}-c\right)^{2}$

There is a minimum value that must be met:

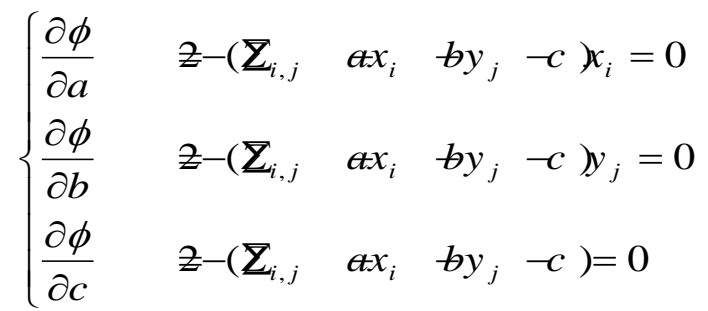

Form of matrix is as follow (where, $\mathrm{N}$ is the groups of the data):

$$
\left[\begin{array}{l}
\sum x z_{z_{i j}} \\
\sum y z_{j, j} \\
\sum z_{i j}
\end{array}\right]=\left[\begin{array}{ccc}
\sum_{i}^{2} x & \sum x_{i} y_{j} & \sum x_{i} \\
\sum x y & \sum y_{j}^{2} & \sum y_{j} \\
\sum x & \sum y_{j} & N
\end{array}\right]\left[\begin{array}{l}
a \\
b \\
c
\end{array}\right]
$$

Input the measured data into formula (1) and then solve it, thus the least squares fitting plane equation can be deduced:

$$
z=0.0009148 x-0.001337 y+1118.761
$$

The flatness error is as follow (where, $\mathrm{F}$ is the flatness error):

$$
F=\max \left(z_{i, j}-z_{i, j}^{*}\right)-\min \left(z_{i, j}-z_{i, j}^{*}\right)
$$

Substituting the measurement data into formula (5), it is easy to calculate the flatness error of the measured sample is $0.48 \mu \mathrm{m}$. It should be noted that the errors include two-dimensional guide mechanism error, the flatness error of the sample itself and the measurement sensor error. 


\section{Conclusions}

In view of the existing difficulties of micro parts in flatness measurement (to reach the sub-micron level), and based on the flexible guiding mechanism, a flatness coordinate measuring device has been designed. It can be seen from the initial test results:

(1)Two dimensional motion platform based on the design of flexible guide mechanism can achieve sub-micron precision in the measurement of plane translation;

(2)The device has the advantages of simple structure, convenient operation, and it is capable of measuring precision parts such as flatness gauge block, air bearing block, aerostatic bearings and other requirements in the submicron range.

The measured sample in the experiment is intercepted from the hard film, so the flatness is unknown, and the test results include the error of the work piece itself, the guide mechanism error and sensor error. In addition, the experimental test of the device is carried out under the conditions of the office, for example there is no constant temperature and vibration isolation and other measures, there also don't have data filtering. Therefore, by improving the experimental environment and the method to test data processing, the measurement accuracy will be further improved.

\section{Acknowledgements}

This work is supported by Shaanxi Science and Technology Department of China, with the supported number No. S2014SZS20-K04.

\section{Reference}

[1] J.L.Yuan, F.H.Zhang and Y.F.Dai. Development Research of Science and Technologies in Ultra-precision Machining Field[J].Journal of Mechanical Engineering, 2010, 46(15): 161-177.

[2] G.Y.Chai, M.Z.Tian and J.Zhou. The Research of the Measurement Method of Flatness Error [J].Machine Tool and Hydraulics, 2007, 35(5): 184-185.

[3] G.Jager, T. Hausotte, E.Manske, H.-J.Buchner, R.Mastylo, N.Dorozhovets, N.Hofmann Nanomeasuring and nanopositionin engineering, Measurement, 2010, 43 (6): 1099-1105.

[4] Eberhard Manske, Gerd Jager, Tino Hausotte and Roland FuBl. Recent developments and challenges of nanopositioning and nanomeasuring technology [J]. Meas. Sci. Technol, 2012, 23(10): 074001-74010.

[5] H.W.Jing, X.Q.Wu, and X.D.Cao,Flatness measurement using precision rotary table[J]. Infrared and Laser Engineering, 2008, 37(4): 141-143.

[6] Y. Liao, A new method for measuring flatness error by using a profiler[J] Metrology \& Measurement Technique, 1998, 10(3): 3-4

[7] L.Y.Wang and S.K.Li,A New Method of Measuring the Flatness Error by Using Cylindricity Measuring Instrument[J].Mechanical Engineering \& Automation, 2007, 145(6): 94-99.

[8] H.Ying: Structure Analysis and Experimental Study of Granite Material Applied in Hydrostatic Guide (MS, Harbin Institute of Technology, China 2008), p. 9.

[9] B.Y.Xiong: Research on structure and control of high precision micro positioner (MS., Northwestern Polytechnical University, China 2005),p.7.

[10]J.J.Yu, State-of-arts of Design Method for Flexure Mechanisms [J].Journal of Mechanical Engineering, 2010, 46(13): 2-9.

[11] S.Y.Li, Study on Micro-motion Mechanism [J]. Aviation Precision ManufacturingTechnology, 2000, 36(4): 6-8. 